\title{
Más allá de la tarea: pistas para una redefinición del concepto de Motivación Escolar
}

Jorge Valenzuela Carreño

Pontificia Universidad Católica de Chile

\section{Resumen}

En el presente trabajo se recogen las distintas aproximaciones al fenómeno de la motivación, y en dicho contexto, se constata y cuestiona aquella conceptualización de la Motivación Escolar que pone su acento en el nivel de la tarea, haciendo de ésta una motivación por realizar ciertas actividades que le son demandadas por la Escuela, pero no necesariamente, una motivación por aprender. En este marco, se proponen pistas sobre algunos elementos o variables que debieran ser considerados en el constructo de la Motivación Escolar. Estas pistas tienen que ver específicamente con la incorporación, dentro del constructo, de los motivos que los alumnos tienen para aprender en la Escuela y que dan sentido a su aprendizaje escolar; y que complementan las variables vinculadas a la tarea. Así, sin olvidar que el alumno debe poner su empeño en realizar las tareas diseñadas para su aprendizaje, debemos volver a mirar las características de la Motivación Escolar, es decir, en aquello que impulsa, conduce y mantiene el esfuerzo del alumno y activa recursos cognitivos para aprender. Aprendizaje que, como es de consenso entre los educadores, no puede limitarse a la mera adquisición de información sino que debe procesada. Así, el conocimiento construido (Pozo, 2003) se hace significativo (Ausubel, 1978) y profundo (Beas, 1994; Beas et al., 2001)

\section{Palabras-clave}

Motivación Escolar - Motivación - Motivación a la tarea. 


\title{
Beyond the task: pointers for a redefinition of the concept of School Motivation
}

Jorge Valenzuela Carreño

Pontificia Universidad Católica de Chile

\begin{abstract}
The work investigates the various approaches to the issue of motivation, and in this context observes and questions the idea of School Motivation that emphasizes the role of tasks, turning the completion of the latter into the motivation to carry out the activities required by the school, instead of stressing the motivation to learn.

The author indicates some aspects or variables that should be considered when building up School Motivation. These pointers are specifically related to the incorporation inside this construct of the reasons pupils have to attend school, and which give meaning to their school learning, thereby complementing the variables associated to the tasks. Thus, without forgetting that the students must put effort into the completion of the tasks designed for their learning, we should recall the essentials of School Motivation, namely, that which drives, conducts and sustains the students' efforts, and triggers cognitive resources to learn. A learning which, as it is consensual among educators, must not limit itself to the mere acquisition of information, but should be processed. The knowledge constructed (Pozo, 2003) is thus made meaningful (Ausubel, 1978) and deep (Beas, 1994; Beas et al., 2001).
\end{abstract}

\section{Keywords}

School motivation - Motivation - Motivation for the task.

Contact:

Jorge Valenzuela

Rue des Sports $2 / 302$

1348, Louvain la Neuve

Belgique

e-mail: valenzu@uc.cl 
Varios son los trabajos que bajo el título motivación al aprendizaje desarrollan la motivación por realizar tareas que, eventualmente, median dicho aprendizaje, pero que no apuntan directamente al querer aprender. Quizás esto se deba a que tal vez en el plano de la tarea sea más fácil la operacionalización de las variables. Con todo, en muchos casos queda la sensación de que para esos autores es evidente que si un alumno está motivado por realizar las actividades escolares, está motivado para aprender. El problema es que esta suposición, lamentablemente, no siempre es correcta.

En vista de lo anterior, en este trabajo queremos cuestionar la conceptualización que normalmente se hace de la Motivación Escolar, y que tiende a reducirla al plano de la tarea, es decir, que la convierte en la motivación por realizar las tareas que la Escuela significa como mediación adecuada para el aprendizaje, y que lamentablemente, no siempre van aparejadas de una motivación por aprender. De hecho es claro que un alumno puede estar muy motivado para realizar una actividad escolar sin activar sus recursos cognitivos para aprender la materia en cuestión.

Por lo anterior presentamos una revisión contextualizada de la "motivación escolar" a partir de las corrientes que han abordado el proceso motivacional, y proponemos pistas para abordar esta noción tomando en consideración el querer aprender como un eje fundamental de la activación de los recursos cognitivos para aprender aquello que la Escuela quiere enseñar a sus alumnos.

\section{Motivación}

La motivación es un constructo hipotético que da cuenta de aquello que impulsa al hombre a actuar (Godefroid 1991; Legendre, 1993; Raynal; Rieunier, 1997). Desde una perspectiva general, los autores están de acuerdo con esta afirmación, mas no necesariamente, con la forma de concebir la naturaleza de la motivación.

Aunque no sea éste el lugar para dar cuenta de todas y cada una de las formas en que se ha abordado el tema motivacional (para una revisión del tema, ver Mateos, 2002), es importante decir algunas palabras sobre la motivación en general, y mostrar en primer lugar, que este constructo aún no siendo del todo homogéneo, es fundamental para entender el accionar del hombre y su aprendizaje intencionado. Esto ha incentivado esfuerzos serios por aportarle claridad conceptual al tema, a partir de cada una de las aproximaciones teóricas que intentan dar cuenta de este fenómeno (Schunk, 1997). Un segundo elemento fundamental a constatar aquí es que el grado de cercanía de las teorías motivacionales al mundo del aprendizaje y de la Escuela también es variable, es decir, varias de estas aproximaciones no han surgido ligadas al mundo escolar, mientras que en otras, la Escuela y su especificidad está en el origen mismo de la pregunta por la motivación y la elaboración de modelos teóricos.

En el caso de aquellas aproximaciones más cercanas al mundo escolar, la gran mayoría tiene en común el hecho de concebir la motivación escolar como una forma de dar cuenta de un fenómeno general en un contexto dado, en nuestro caso, la Escuela. Por ello, ha dirigido su mirada hacia el desempeño escolar teniendo como foco la motivación de los alumnos hacia la tarea (task, tâche). En este sentido, a estos autores les interesa fundamentalmente entender cuáles son los factores y relaciones pertinentes, para explicar el hecho de que un alumno se aboque a realizar las tareas que la Escuela les propone como mediación del aprendizaje.

A partir de los trabajos de Atkinson (1957) se ha desarrollado una línea de investigación que intenta establecer aquello que afecta a la necesidad de logro que todo ser humano posee, máxime, en un contexto como el escolar. Esta perspectiva se ha visto desarrollada y complementada por otras teorías y modelos tales como el de la Atribución Causal de Bernard Weiner (1986a; b) y los modelos de Expectación/Valor (Expectancy and Value) de Allan Wigfield y Jacquelynne Eccles (1992; 2000); Eccles et al. (1983); Wigfield (1994). 
Algunos aportes provienen desde la perspectiva atribucional. Autores como Weiner (1986a) nos plantean que los estudiantes evalúan su éxito o fracaso atribuyéndole causas. Según este autor, el tipo de atribuciones que el alumno realiza es determinante para poner en juego su esfuerzo y su compromiso con la tarea. En otras palabras, unos determinados tipos de atribuciones repercuten más positivamente sobre el deseo de tener éxito en las tareas. Estas atribuciones se distinguen bajo los criterios de locus de causalidad, estabilidad y controlabilidad y dan lugar a determinadas emociones que, finalmente, tienen un impacto importante en la motivación del alumno.

Otro aporte a la explicación de la motivación de logro (achievement goals) proviene de Eccles y Wigfield (2002), Wigfield y Eccles $(1992 ; 2004)$ quienes plantean que la motivación depende básicamente del valor percibido y asignado a la tarea y de la expectativa que la persona tiene de realizarla con éxito. El así llamado modelo de Expectancy and Value (Eccles et al., 1983; Wigfield, 1994) afirma que en la motivación final depende del producto de estos dos factores (E $x \mathrm{~V}$ ). Dicho de otra manera, los teóricos en esta tradición argumentan que la elección individual, persistencia y resultados referidos a una tarea pueden ser explicados por sus creencias sobre cuán bien ellos harán la actividad, por una parte, y el grado en que ellos valoran dicha actividad, por otro (Atkinson, 1957; Eccles et al., 1983; Wigfield, 1994; Wigfield; Eccles, 1992), valoración, que se operacionaliza a partir de 4 componentes: el interés, la importancia, costo y la utilidad de la tarea (cf. Eccles; Wigfield, 2002; Neuville, 2004; Neuville, Bourgeois; Frenay, 2007).

Complementariamente, en el marco de esta perspectiva, encontramos los aportes de Deci y Ryan, quienes a partir de la distinción entre motivación intrínseca y extrínseca nos muestran cómo la primera afecta más positivamente el desempeño escolar (Deci; Ryan, 1985; 1999; Sansone; Harackiewicz, 2000). Es decir, cuando los motivos para realizar las tareas es- colares son inherentemente interesantes o apetecibles (Deci; Koestner; Ryan, 2001) el afecto por la tarea es mayor y conduce a mejores resultados.

Asociado con lo anterior, Deci y Ryan (1985) y Deci et al. (1991) entienden que la motivación a la tarea mejora en función de la percepción que el alumno tiene del control sobre su tarea (self-determination). En la medida en que el alumno siente que la tarea es SU elección y no algo impuesto, la motivación aumenta y con ello las posibilidades de obtener un mejor rendimiento (Ryan; Deci, 2000).

Pero las percepciones involucradas en la motivación no se limitan al grado de autonomía frente a la tarea. Bandura (1977, 1982, 1986, 1988, 1989) y Bandura et al. (1996) ha puesto de relieve la importancia de la Autoeficacia. Este constructo refiere a los juicios personales sobre la propia capacidad para organizar y ejecutar cursos de acción en función de alcanzar un determinado objetivo (Zimmerman, 2000), y por tanto, facilita o restringe el compromiso con la tarea. De esta manera, un alumno con alta autoeficacia creerá que tiene las capacidades suficientes para realizar una tarea mientras que uno con baja autoeficacia, al dudar de sus capacidades, se autolimita poniendo en tela de juicio sus posibilidades de realizarla.

Otra forma de encarar el tema es aquella que distingue los tipos de metas que los estudiantes ponen en juego frente a la tarea escolar (goals theories). En esta perspectiva, son variadas las clasificaciones que se han propuesto, por de pronto la distinción intrínseco/ extrínseco de Deci y Ryan, pero hay otras... Veamos algunas:

Heredando en parte la tradición de Maslow quien proponía una jerarquía de necesidades, Martin Ford (1992) propone una clasificación de motivos que impulsarían, a manera de causa final, el actuar humano. Para Ford (1992), la motivación es el producto de las metas, las emociones y las creencias de las propias competencias (personal agency beliefs). En el caso de este autor, estos motivos (personal goals) no están 
jerarquizados como en el caso de Maslow, sino que se refieren a ámbitos o dominios en el cual el hombre se desenvuelve.

El aporte de Ford, pese a estar referido a la motivación en general, nos da pistas para pensar la motivación específica para aprender en la Escuela, la cual a nuestro juicio no debe reducirse a una motivación instrumental a la tarea (hacer las actividades que los maestros proponen como mediación del aprendizajes) sino que debe considerar. Asimismo, un plano más general de motivos, que den sentido a aprender lo que la Escuela propone como aprendizaje.

Otro aporte importante al estudio de la motivación, corresponde al realizado por las psicólogas Dweck y Leggett (1988) las que proponen distinguir metas de aprendizaje y metas de ejecución. En el primer caso, el alumno se propone como objetivo aprender, dominar una competencia, disfrutar (enjoy themselves) o sentirse competentes (Byrnes, 1996) mientras que en el segundo lo que importa es rendir adecuadamente frente a la tarea (obtener buenas calificaciones), lo que generalmente viene aparejado con el deseo de "ganar la aprobación de los otros, mostrarse mejores que sus pares, a obtener recompensas o evitar castigos” (Byrnes, 1996, p. 230). La investigación (Dweck, 1986; Nicholls, 1983; Wentzel; 1991) ha mostrado que los alumnos que tienen metas de aprendizaje abordan de manera más profunda el quehacer escolar lo que reditúa, finalmente, en mejores niveles de desempeño que aquellos que buscan fundamentalmente realizar la tarea y nada más.

Otra forma de distinguir las metas de los estudiantes para dar cuenta de la motivación a la tarea es distinguir las Metas Académicas de las Metas Sociales (academic v/s social goals). Las primeras, se orientan a la tarea directamente hacia metas de carácter académico tales como aprender cosas nuevas, comprender los contenidos propuestos o realizar las actividades en el tiempo dispuesto para ello. La segunda, orientada hacia los motivos sociales, se refieren a comportamientos tales como lograr la aprobación de otros, entablar amistad con compañeros, entretenerse (Byrnes, 1996). Para Wentzel (1991), es necesario orientar estos últimos hacia un horizonte de metas académicas, ya que estas últimas están más positivamente asociadas a un mejor rendimiento.

Por otra parte, con una preocupación más práctica, otro grupo de autores han construido modelos que intentan contribuir a la mejora del rendimiento escolar tomando como punto de partida los aspectos motivacionales del alumno.

Para ello, han recogido e integrado los aportes y desarrollos en el campo de la investigación motivacional que tiene un interés fundamentalmente explicativo de la motivación. Es el caso, por ejemplo, de Roland Viau (1997) quien define la motivación en contexto escolar como "un estado dinámico que tiene sus orígenes en las percepciones que el alumno tiene de sí mismo y de su medio y que lo incita a elegir una actividad, a comprometerse y a perseverar en ella a fin de alcanzar un objetivo" (Viau, 1997, p. 7). En este modelo marcadamente socio-cognitivista, se recogen los aportes anteriores, entre ellos, como subraya Tierry Huart (2001) el de la interacción recíproca propuesta por Bandura (1978) o los motivos intrínsecos y extrínsecos (Deci; Ryan, 1985), entre otros.

También dentro de la tradición francófona, que se pregunta por la motivación para explicar el fracaso escolar (échec scolaire), queremos hacer referencia al trabajo de Jacques Tardif (1992), para quien la motivación depende de dos sistemas de concepciones y de tres sistemas de percepciones. Las concepciones están referidas a los objetivos de la escuela. Para este profesor de la Universidad de Sherbrooke lo fundamental estriba en el reconocimiento de la escuela como una instancia cuyo objetivo es el aprendizaje o la evaluación y sería esta concepción la que estaría a la base de las metas de aprendizaje o de ejecución (Dweck; Leggett, 1988). Una segunda concepción que incidiría en al motivación es la de la inteligencia (ver también, Braten; Strømsø, 2004; Dupeyrat; Mariné, 2005). En la medida que el alumno concibe la inteligencia como algo dado, ina- 
movible, limita las posibilidades de superación y de mejores desempeños que los actuales, mientras que si la concibe como algo dinámico, modificable o desarrollable da espacio para que el esfuerzo personal actúe y consiga mejorar sus aprendizajes. Por su parte, los tres sistemas de percepción propuestos por Tardif (1992) coinciden con posturas ya presentadas. La percepción del valor de la tarea (value), de la dificultad de ella (expectancy y autoeficacia) y de controlabilidad, emparentándose con la propuesta de la autodeterminación de Ryan y Deci y con los aportes de Weiner.

La tradición académica hispana ha hecho por cierto lo suyo. Desde una sensibilidad más cercana a la Escuela, autores como José Antonio Huertas (1997), Jesús Alonso Tapia (1997; 2002) entre otros, han recogido estos aportes y los han releído desde la compleja dinámica escolar buscando estrategias pedagógicas que estimulen una mayor motivación por parte de los alumnos hacia la tarea.

Pero en esta mar de teorias, aproximaciones y aportes ¿qué vamos a entender por Motivación Escolar? ¿Cómo nos aproximamos a la motivación por aprender en la Escuela?

\section{¿Motivación en contexto escolar o Motivación Escolar?}

Las perspectivas anteriormente señaladas cuando son aplicadas en el ámbito de la educación escolar, tienden, en general, a efectuar un proceso de aplicación de la teoría general al ámbito de la Escuela (Motivación en contexto escolar). Al hacer esto centran su atención en las acciones que realiza el estudiante en este contexto, a saber: realizar las actividades o tareas que la Escuela le propone como mediación de su aprendizaje o, como diría Tardif (1992) la motivación [en contexto] escolar concierne el compromiso, la participación y la persistencia de alumno en las actividades de la clase.

Ciertamente, la motivación a la tarea es un aspecto importantísimo en la consecución de los logros académicos y la literatura es abundante en mostrar cómo a mayor motivación hacia la tarea se observan mejores resultados académicos (Willingham; Polack; Lewis, 2002; Schiefele; Krapp; Winteler, 1992; Tobías, 1994; entre otros).

No obstante lo anterior, existe una dificultad no menor. Hay alumnos que pueden estar muy motivados por realizar las tareas propuestas por la Escuela y no querer aprender, o al menos, no de manera significativa (Ausubel, 1978) y profunda (Beas, 1994; Beas et al., 2001).

Entre los educadores hay consenso en que no basta con la adquisición de información, es necesario que dicha información sea procesada, vinculada con conocimientos anteriores, en fin, que exista construcción del conocimiento (Pozo, 2003). En esta perspectiva, querer realizar la tarea escolar no es sinónimo de querer aprender de manera significativa y profunda, de querer activar los recursos cognitivos para procesar la información disponible.

Nos parece que, sin olvidar que el alumno debe poner su empeño en realizar las tareas diseñadas para su aprendizaje, debemos enfocarnos en las características de la Motivación Escolar, es decir en aquello que impulsa, conduce, mantiene el esfuerzo y activa recursos cognitivos para aprender.

Este aprender tiene relación con un contexto concreto. Se trata de aprender una serie de contenidos que NO han sido elegidos por los alumnos y NO son optativos. Los contenidos escolares son fundamentalmente decididos por el sistema escolar como pertinente para la formación de las generaciones jóvenes. Es la Escuela quien decide cuáles son los contenidos culturales relevantes y cuáles no.

En este contexto, es claro que se puede querer realizar la tarea, en cualquiera de sus grados. Pero, ¿por qué el alumno querría aprender lo que la Escuela quiere enseñarle? ¿Por qué activaría sus recursos cognitivos para aprender significativamente? 0 dicho de otro modo: ¿qué da sentido a aprender en la Escuela?

Para poder dar cuenta de estas dificultades queremos proponer una visión de la Motivación 
Escolar que toma en consideración los aportes que ven la motivación desde la tarea, pero que integra además elementos de sentido que hacen posible que la activación de recursos cognitivos para aprender en la Escuela tenga un marco referencial que haga posible que los aprendizajes devengan significativos. Dicho de otro modo, queremos mirar la motivación desde el aprendizaje profundo, desde el aprendizaje que implica la construcción de conocimiento significativo.

\section{Motivación Escolar}

En este contexto, se hace necesario explicitar algunos supuestos, opciones y aportes teóricos que nos parecen relevantes para fundamentar y dar mayor comprensión a esta propuesta. Ellos tienen que ver, fundamentalmente con querer aprender.

Recordando nuevamente que nos situamos en el plano de la Escuela, un primer elemento a tener en consideración es que es condición necesaria para que exista aprendizaje explícito la presencia de tres elementos (ver Figura 1). En primer lugar, ser capaz de aprender, es decir, tener las posibilidades orgánicas e intelectuales para enfrentar el proceso de aprendizaje (por ejemplo, es evidente que una persona con daño neurológico severo no puede aprender todo lo que la Escuela espera). En segundo lugar, es necesario que exista la oportunidad de aprendizaje. Evidentemente, no basta con ser capaz de aprender, es necesario que se den las instancias correspondientes para que el aprendizaje ocurra: acceso a los recursos de aprendizaje, metodología apropiada, clima de aula adecuado, etc. Finalmente, junto con la capacidad y las oportunidades de aprendizaje, es necesario que el alumno quiera aprender (Parmentier, 1999).

\section{Querer aprender}

El querer (aprender) implica, de alguna manera, activar y mantener en actividad una serie de recursos cognitivos, tales como la atención, memoria, y procesos psicológicos superiores para poder adquirir y procesar información, desarrollar ciertas destrezas y comprometerse en tareas que impliquen pensar (cf. Tishman; Jay; Perkins, 1993).

Figura 1: Requisitos para aprender de manera explícita.

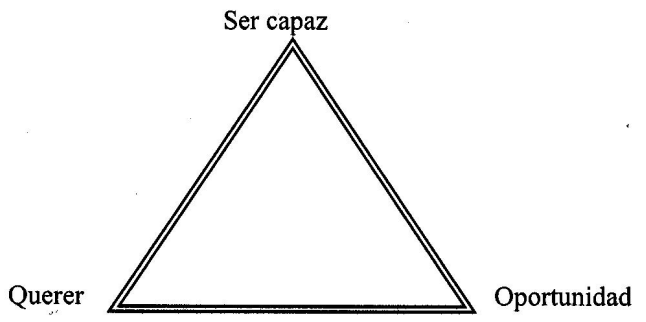

Pero ¿porqué la gente quiere algo? Dicho de un modo simple, queremos algo cuando lo "necesitamos", en lenguaje de Joseph Nuttin (1980)', cuando se da una relación requerida (rélation réquise) entre el sujeto y el objeto. Queremos aprender cuando visualizamos de alguna manera que dicho aprendizaje tiene sentido, es decir, cuando "tiende a, se orienta a, sirve para, significa algo" (Marquínez, 1980 p. 142). Ahora bien, el objeto, en cuanto tal, es una construcción que realizamos a partir de las posibilidades que nos otorga su realidad material (Zubiri, 1962; Pintor-Ramos, 1993). Así, el objeto es una cosa a la cual hemos atribuido sentido transformándola en una cosa-sentido.

Pero el sujeto (el polo individuo, en el lenguaje de Nuttin) que se relaciona con este objeto y establece relaciones requeridas, también es, de alguna manera, una construcción. En esta línea, y siguiendo al Heidegger (1971) de Ser y Tiempo, podemos afirmar que el sujeto se autointerpreta en cada acto de interpretación del mundo, en cada asignación de sentido. Este es el otro polo de esta relación. De esta manera,

1. En Nuttin el carácter requerido de ciertas relaciones se manifiesta por insuficiencias funcionales (carencias) y por otra parte, por las respuestas de búsqueda, de refuerzo y de aceptación afectiva (placer), presentándose el carácter requerido con diversos grados de urgencia y de importancia para el funcionamiento óptimo del individuo. 
la relación "sujeto-objeto" (como ámbito genético de la motivación) es una relación entre un sujeto en continua reinterpretación y una cosa a la que se le asignan unos determinados sentidos. Por lo tanto, las creencias sobre el objeto y sobre sí mismo, que tenga el sujeto, son fundamentales para el nacimiento de la motivación y para entender porqué las concepciones y representaciones que tengamos de nosotros mismos son determinantes para entender esta tensión motivacional con un objeto. La manera en que nos autoconceptualizamos y auto-percibimos son el cristal a través del cual miramos (y reconstruimos constantemente) los objetos. De esto, por ejemplo, depende en parte, la valorización que hagamos del objeto, o la percepción de dificultad que le asignemos a una tarea.

Por otra parte aparece el objeto de ese querer: el aprendizaje.

En primer lugar, puntualicemos que nos referimos a un aprendizaje en la Escuela, y por tanto, con un claro carácter disciplinario (García, 1997), explícito e intencionado desde el sistema escolar (Ainzencang, 2001; Elichiry, 2001). Idealmente, este aprendizaje escolar debe ser un Aprendizaje Profundo (Beas et al., 1997; 1996; 2001) por tanto, no se trata de un simple cambio de conducta o la adquisición de nueva información. El Aprendizaje Profundo implica la construcción de conocimiento a través del establecimiento de una red de conexiones significativas, tanto dentro de la disciplina, como en su relación con otros dominios de conocimiento $y$, por supuesto, también con la vida diaria, pero para tener un aprendizaje significativo no es suficiente con disponer de un material potencialmente significativo y de ideas pertinentes en las estructuras cognitivas, a las cuales relacionarlas, es necesario también tener una disposición a aprender de manera significativa. Ciertamente, este componente, de igual forma que los anteriores, es requisito sine qua non para un aprendizaje significativo (Ausubel, 1978; Valenzuela, 2002).

En síntesis, querer aprender significa, de alguna manera, necesitar ese aprendizaje. Y, como veremos un poco más adelante, existen diversas formas o motivos para querer aprender en la escuela. Estas "razones" o motivos para querer aprender en la Escuela dan sentido a la actividad escolar y por tanto posibilitan el carácter querible del contenido escolar en la medida que sirve para, se orienta a, tiende a, ...en la medida que tiene sentido. Este sentido marca la lógica bajo la cual el alumno se enfrenta a un contenido escolar que no ha sido definido por él, sino por la institución escolar.

A partir de lo anterior, podriamos visualizar ciertos elementos que ha de tenerse en consideración a la hora de configurar los factores de la motivación escolar. Recordemos que casi todos los elementos tienen historia y el respaldo de investigaciones que vinculan cada uno de los constructos con el desempeño académico en la Escuela. En concreto, resulta claro que debemos tomar en consideración las percepciones y concepciones que el sujeto hace de sí mismo y del contexto general donde se inserta la actividad escolar. De igual manera, no podemos obviar las percepciones sobre la tarea y sobre la relación que el sujeto establece con ella.

Por otra parte no es posible olvidar el aspecto emocional. Las emociones tienen un gran rol dentro de la dinámica motivacional (Turner; Meyer; Schweinle, 2003), ellos son a la vez indicadores y retroalimentación de cómo marcha esta relación con el objeto.

\section{Niveles de análisis de la actividad escolar}

Hagamos un último alcance antes de analizar con más detalles cada uno de los elementos que rescatamos como significativos en la Motivación Escolar. Creemos importante distinguir niveles de análisis cuando abordamos el tema motivacional. Como ya hemos sugerido y explicitaremos con más detalle a continuación, los elementos o factores de la Motivación escolar podemos situarlos en dos niveles distintos. Un nivel general que tiene que ver con la actividad escolar en su conjunto y un segundo nivel que tiene que ver con la tarea, es decir, 
con el conjunto de demandas y mediaciones que la Escuela propone con vistas a cumplir con los objetivos de aprendizaje.

Para distinguir estos dos niveles de análisis nos inspiramos en la distinción de Leontiev sobre los niveles de la actividad (Leontiev, 1978; Glassman, 1996; Kozulin, 1986; Rochex, 1998). En Leontiev la actividad se desarrolla como un esquema en tres niveles. Estos niveles consideran la fuerza motivacional de la actividad general (motivos), los propósitos directos de la acción individual manifestados como objetivos o metas y los métodos externos de uso individual para realizar estos objetivos, las operaciones (Glassman, 1996) y cada uno de estos niveles cumple una función distinta. Los motivos (motive, motifs) realizan la función de incitación, los objetivos o metas, la de la orientación, y finalmente, los modos operatorios cumplen la función de realización (ver Figura 2).

Figura 2: Niveles de la actividad en Leontiev.

\begin{tabular}{|c|c|c|}
\hline NIVELES & REGULADOR & FUNCIÓN \\
\hline De la Actividad & Los motivos & $\longrightarrow$ de incitación \\
\hline De la Acción & Los objetivos o metas & $\longrightarrow$ de orientación \\
\hline De las operac & Los modos operatorios & $\longrightarrow$ de realización \\
\hline
\end{tabular}

En esta línea, el nivel de la actividad, la cual es regulada por los motivos, depende de cómo nos representamos el mundo, a nosotros mismos y la actividad concreta en relación con los dos primeros. Por ello, dentro de este primer nivel situamos los elementos motivacionales que tienen que ver con la actividad general de aprender en la escuela, en concreto: las representaciones que tenemos sobre los objetivos de la Escuela, el sentido atribuido a aprender lo que la escuela quiere enseñar, las representaciones que tenemos sobre nuestra capacidad para enfrentar la actividad escolar y la concepción de inteligencia.

En un segundo nivel de análisis aparecen los elementos vinculados con lo que Leontiev llama el nivel de la acción y que estaría regulado por las metas, y que tiene que ver con las tareas concre- tas que se demandan a los estudiantes en el proceso escolar, por lo que la designaremos con el nombre de nivel de la tarea. En concreto, nos referimos a los tipos de metas que el alumno se propone frente a la tarea, las percepciones sobre el valor, la dificultad y el control de dichas tareas y las atribuciones causales que realizamos sobre los resultados obtenidos en ellas.

Si bien es cierto que Leontiev plantea tres niveles, nuestro análisis de la motivación concierne a los dos primeros, por lo que no abordaremos el nivel de las operaciones en este momento.

Pero veamos cada uno de estos elementos con más detalle y cómo se relacionan entre sí.

\section{Factores y elementos en la Motivación Escolar}

Podríamos de decir con Archambault y Chouinard (1996) que la Motivación Escolar es el conjunto de determinantes que impulsan al alumno a comprometerse activamente en su proceso de aprendizaje, a adoptar comportamientos susceptibles de conducir a la realización de los objetivos de aprendizaje que él persigue y a perseverar ante las dificultades. ¿Pero cuáles son esos elementos o factores? ¿Qué elementos ayudan o dificultan el que un alumno quiera aprender aquello que le es propuesto por la Escuela? En este análisis de los factores motivacionales seguiremos de cerca la propuesta que hace Jacques Tardif, aun cuando integremos otros elementos.

\section{Factores y elementos a considerar en el ámbito de la actividad}

\section{Concepciones sobre el objetivo de la Escuela}

Según Jacques Tardif (1992) uno de los componentes de la motivación es la concepción por parte del alumno de los objetivos perseguidos por la Escuela (Figura 3). Según este autor, esta sería la matriz a partir de la cual los estudiantes se 
proponen metas de aprendizaje (learning goals) o de logro o ejecución (performance goals) que han sido estudiadas entre otros por Dwek y leggett (1988) o Nicholls (1983).

Para Tardif, el reconocimiento de los objetivos perseguidos por la Escuela (de aprendizaje/ de Evaluación) sitúa al estudiante de una manera particular frente al quehacer académico escolar, incidiendo en la manera en cómo concibe el éxito escolar (aprender o lograr calificaciones interpretadas en clave de comparación con el resto), el nivel de compromiso en las actividades (en función de un desarrollo personal o en función de la protección de su autoestima), el poder sobre la actividad, el grado de participación en la actividad, y las atribuciones que realiza sobre los resultados (Tardif, 1992).

Aunque aparentemente la perspectiva de Tardif coincide con las teorizaciones sobre tipos de metas, este autor introduce un matiz importante. No se trata sólo del tipo de metas que tiene el alumno, sino de destacar el papel de la escuela en la formación de estas metas. Si nos fijamos bien las concepciones son el marco que posibilita, finalmente, que el alumno adopte metas en uno $u$ otro sentido.

\section{Sentido del aprendizaje escolar}

El sentido atribuido a aprender lo que la Escuela quiere enseñar o Sentido del Aprendizaje Escolar (ver Figura 3) aparece como un segundo elemento a tener en consideración. En la medida en que la lógica bajo la cual el alumno interpreta la realidad escolar y le da sentido a aprender coincide con la de su profesor, hay posibilidades de que la propuesta escolar pueda tener un sentido potente para el alumno. De esta manera, la concordancia entre el sentido atribuido a aprender en la Escuela, dado por del alumno, y la lógica bajo la cual se intencionan los contenidos escolares por parte del profesor, hace posible que el estudiante quiera establecer conexiones significativas entre lo que está aprendiendo y sus conocimientos previos, es decir, a mayor consonancia entre estas dos lógicas, mayores

Figura 3: Elementos y factores que inciden en la Motivación Escolar.

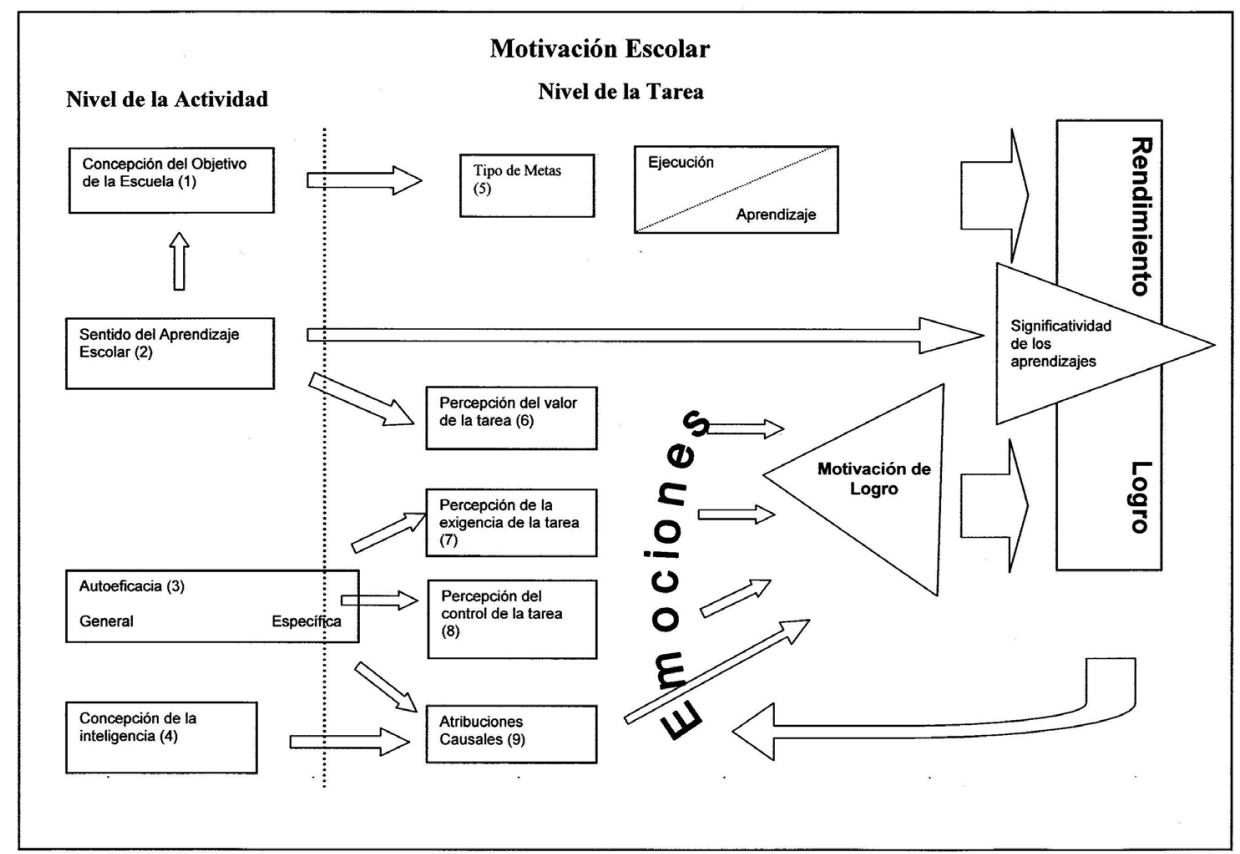


son las posibilidades de que aprender lo que la Escuela quiere enseñar tenga sentido, sea significativo y profundo.

Ahora bien, la forma en la cual los estudiantes valoran los distintos motivos para aprender en la escuela no es un acto meramente individual ya que sus valoraciones estarían mediadas por la "validación" de sus pares. De esta manera, en la medida en que la estructura valorativa del alumno sea validada por sus pares, se incrementaría la fuerza motivacional de ese motivo. $Y$ a la inversa, si las razones por las cuales un alumno quiere aprender en la Escuela son descalificadas, implícita o explícitamente, por sus pares, probablemente tendrán una potencia menor que si estuviese en un contexto que valide dicho motivo.

Evidentemente, el sentido atribuido a aprender en la Escuela se relaciona con la forma en la cual concebimos la funcionalidad de la institución escolar y los objetivos perseguidos por ella, pero incide también en la asignación de valor a la tarea.

\section{Autoeficacia}

Un tercer elemento a tener en consideración es la Autoeficacia, es decir los juicios que las personas realizan acerca de sus propias capacidades para desempeñarse en un dominio dado (ver Figura 1), donde la concepción resultante de estos juicios puede poner, por así decirlo, (auto-)barreras a las posibilidades de desempeño en el ámbito escolar. Al eliminar estas trabas autoimpuestas e incrementar el sentimiento de autoeficacia, se abre camino para que la motivación pueda concretarse en mejores desempeños.

Este constructo, propuesto por Albert Bandura (1977) se relaciona a su vez con otros dos elementos considerados aquí; en primer lugar, con la percepción de exigencia de la tarea, es decir, con la dificultad que la persona le atribuye la tarea, y en segundo término, con la percepción del control que se ejerce sobre ella.

\section{Concepciones sobre la inteligencia}

Otro elemento relevante a tener en consideración para dar cuenta de la motivación, es la concepción que tienen los alumnos acerca de la inteligencia (ver Figura 1). La investigación ha mostrado cómo las creencias y teorías implícitas sobre la inteligencia juegan un rol importante en la disposición al aprendizaje (Braten; Strømsø, 2004)

En general, podemos distinguir dos tipos de concepciones de inteligencia. La primera, la considera una entidad estable, fija, que no evoluciona con el tiempo. Es la representación que tenemos asociada a los modelos psicométricos y al coeficiente intelectual (1Q). La segunda, por el contrario, la concibe como desarrollable, móvil, que puede evolucionar con el correr del tiempo. En este caso, la inteligencia es la resultante de un conjunto de conocimientos y de estrategias tanto cognitivas como metacognitivas susceptibles de desarrollarse y que mejoran la capacidad de actuar "inteligentemente" en relación con el medio (para una revisión del concepto véase Rosas; Boetto; Jordan, 1999).

Estudios muestran que la segunda concepción es frecuente en los primeros años de escolarización pero, a medida que pasa el tiempo, cede paso a la visión estática e innatista de la inteligencia (Tardif, 1992).

La concepción de inteligencia genera consecuencias múltiples. Los niveles de confianza dependen, en gran medida, de si conciben la inteligencia como algo fijo o algo flexible (Cain; Dweck, 1995). Los alumnos que la conciben como estrategias evolutivas tienden a mostrar objetivos de aprendizaje relacionados con la construcción del conocimiento, eligen aquellas actividades que contribuyen a incrementar sus conocimientos y optan por aquellas que aparecen como desafiantes. Esta forma de concebir la inteligencia contribuye a que los alumnos asuman que los errores y dificultades de las tareas no cuestionan su inteligencia y a que estén abiertos a aprender de sus errores y a revisar sus estrategias. Por el contrario, aquellos 
alumnos que conciben la inteligencia como una entidad estable, tienden a tener metas asociadas con la evaluación, y la validación de sus conocimientos; asimismo, seleccionan actividades que contribuyan a validar su competencia y que representen el mínimo riesgo posible a este respecto (Tardif, 1992).

\section{Factores y elementos a considerar en el ámbito de la Tarea}

\section{Tipos de metas}

Los tipos de metas son una concreción o equivalente, a nivel de la tarea, de lo que ya hemos tratado en el apartado sobre la concepción sobre los objetivos de la Escuela y por tanto, en buena medida, interdependiente con ella. Por otra parte, el tipo de metas depende, en algún grado, de las creencias y teorías implícitas que se tienen sobre la inteligencia (Braten; Strømsø, 2004).

Varios son los autores que han estudiado la relación existente entre el tipo de objetivos que el alumno se plantea frente a las actividades escolares (ver Figura 1) y el rendimiento académico a través las estrategias cognitivas y autoregultorias (Suarez; González; Valle, 2001). Pero quizás una de las distinciones que más ha ayudado en este sentido es la que proponen Dweck y legett (1988) quienes distinguen entre las metas de aprendizaje y las de ejecución (Learning goals/ Performance goals).

Los alumnos que enfrentan sus tareas escolares bajo la lógica de metas de aprendizaje muestran una serie de conductas que finalmente los lleva a obtener mejores rendimientos que aquellos alumnos que tenían metas de ejecución.

La razón de lo anterior es que estos alumnos al tener metas de aprendizaje tiene más sentido para ellos el desarrollar mejores estrategias cognitivas y metacognitivas (Somuncuoglu; Yildirim, 1999).

En síntesis, la manera en cómo abordamos la tarea escolar, como algo que debemos hacer en pos de aprender o en función de la cual el alumno será evaluado, lo predispone para actuar en consecuencia: activar una serie de recursos cognitivos y de estrategias para establecer relaciones significativas y lograr una comprensión profunda o, en el segundo caso, para desplegar las estrategias necesarias para obtener una calificación que él considere adecuada, buena o suficiente.

\section{Percepción del valor de la tarea}

Como ya habíamos dicho, uno de los elementos fundamentales de la motivación de logro se estructura en función del valor que asignamos a la tarea (Eccles; Wigfield, 2002). En la medida en que la tarea tiene sentido y la valoramos positivamente (ver Figura 3) existen mayores posibilidades de querer activar los recursos cognitivos y metacognitivos de los que disponemos para realizarla. El valor de la tarea se compone de la funcionalidad que ella tiene y de los "ecos" personales, sociales o profesionales que ella genera.

Si el alumno percibe que una tarea determinada tiene "ecos" importantes o útiles a nivel personal o social, que ella conduce a la adquisición de conocimientos utilizables fuera del ámbito de la clase, el alumno estaría dispuesto a invertir en energía y esfuerzo para realizar ese tipo de actividad. Por el contrario, si el alumno no percibe su importancia es probable que los niveles de atención, por ejemplo, sean bastante menos altos, y que su compromiso con dicha tarea sea más bien débil (Tardif, 1992).

\section{Percepción sobre las exigencias de la tarea}

Otro elemento a considerar aquí es que, junto con asignarle valor a la tarea, el alumno toma en consideración las exigencias que ésta demanda (ver Figura 3). El alumno realiza un juicio metacognitivo sobre los conocimientos previos de que dispone sobre el particular, las estrategias necesarias para llevar a cabo dicha tarea adecuadamente, las etapas que implica la realización de dicha tarea $\mathrm{y}$, finalmente, los 
criterios a partir de los cuales este alumno define qué significa realizar exitosamente la tarea.

En la medida en que el alumno se percibe a sí mismo como poseedor de los conocimientos Básicos (esquemas previos) adecuados o suficientes para emprender la tarea, que percibe que cuenta con estrategias que le permitirán cumplir con ella, que evalúa (Perkins, 1992) las etapas que implica la tarea (tiempo, esfuerzo, etc.), y que tiene una idea clara de qué significa hacer bien la tarea (cumplir, aparentar cumplir, aprender, comprender profundamente, etc.) ...variará la disposición del alumno y su nivel de compromiso con la tarea.

\section{Percepción el control sobre la tarea}

Junto con los dos elementos anteriormente enunciados, el compromiso con la tarea depende, en este caso, muy importantemente, de la percepción de control que el alumno tiene sobre la tarea (ver Figura 3). Desde los años 80 sabemos que la motivación a la tarea está fuertemente influenciada por la percepción de eficacia y de control de aquello que debe poner en juego para tener éxito en la tarea (Bandura 1982; Locke; Zubritzky; Lee, 1982, en Tardif, 1992; Shunck, 1984).

Una de las implicancias mayores de la percepción de control sobre la tarea en contexto escolar, como lo demuestra Watkins (1984, en Tardif, 1992), es que en la medida que el alumno percibe que tiene control sobre ella, existe más tendencia a tratar de forma significativa la información que en el caso contrario. Por otra parte, sus estudios muestran que los alumnos que perciben una falta de control sobre la tarea tienden a tratarlas mecánicamente como tareas de memorización o a considerar las distintas partes de la tarea como elementos autónomos y, obviamente, a desarrollar estrategias defensivas que protejan al alumno ante la incertidumbre de la tarea.

Finalmente, la investigación ha mostrado reiteradamente que los logros escolares están asociados de manera positiva y estrecha con las percepciones que el alumno tiene de sí mismo y del control percibido sobre la tarea (ver los meta-análisis de Findley; Cooper, 1983; Hansford; Hattie, 1982).

\section{Atribuciones causales}

Las atribuciones causales sobre el éxito o fracaso en la realización de la tarea son fuente, asimismo de la percepción de control que un alumno pueda tener sobre la tarea (ver Figura 3).

Según este modelo, una secuencia motivacional se inicia con un resultado que la persona interpreta como éxito (meta alcanzada) o fracaso (meta no alcanzada) y relaciona primariamente con sentimientos de felicidad y tristeza/frustración. Si el resultado es inesperado, negativo o importante, el sujeto busca la causa de tal resultado, teniendo en cuenta los diversos antecedentes de información (historia personal pasada, ejecución de otros), las reglas causales, la perspectiva actor/observador, los sesgos atribucionales, etc., que culmina en la decisión de atribuir el resultado a una causa singular. Las causas singulares se diferencian y se parecen en determinadas propiedades básicas subyacentes a todas ellas, denominadas dimensiones causales, que permiten compararlas y contrastarlas cuantitativamente (Manassero; Vásquez, 2000).

Weiner nos plantea que en la medida que las atribuciones sobre el resultado de la tarea tengan un locus interno, sean inestables y controlables, hay mayores posibilidades de compromiso con la tarea. Desde esta perspectiva, la Motivación de Logro, (y a través de ella, el rendimiento académico), se ve favorecida en la medida que las atribuciones causales sean:

1. de carácter interno, es decir que se atribuye a uno mismo la responsabilidad por el éxito o fracaso de la meta. Ejemplo de lo anterior lo constituye la atribución a la habilidad (carácter interno) mientras que la dificultad de la tarea adquiriría la connotación opuesta (carácter externo).

2. de carácter inestable, es decir que aquello que causa el éxito o el fracaso es susceptible 
de modificación. El tipo de atribución inestable varía, a su vez dependiendo si es de carácter interno o externo (ver Figura 4). Con todo, es la atribución al esfuerzo la que más claramente facilita el aprendizaje. Otras atribuciones inestables como la suerte, si bien puede variar, no es controlable, y en muchos casos se vuelve una atribución estable (Bueno, 1993; 2004). Eventualmente, la habilidad o la inteligencia generalmente concebida como estable, pueden también ser concebidas como algo desarrollables facilitando de igual manera la Motivación de Logro.

3. de carácter controlable, es decir que el sujeto conciba atribuciones cuya naturaleza sea controlable por él. Ejemplo de lo anterior es el esfuerzo, el cual puede verse afectado por voluntad del sujeto, mientras que la suerte escapa a cualquier posibilidad de control.

Dependiendo de la atribución que el aprendiz genere en torno a las causas que producen una actuación eficaz o ineficaz, establece una serie de conceptos, de expectativas, que condicionan la actividad a la hora de iniciar una nueva tarea (Ugartetxea, 2002). De esta manera, la explicación que un alumno se da sobre el resultado de una tarea y su clasificación atribucional, determina en gran medida, su posterior aprendizaje (cf. Alonso Tapia, 1984; 1987; Manassero; Vásquez, 1996; 2000).

Ahora bien, dentro de la teoría de Bernard Weiner las atribuciones están asociadas a unas determinadas emociones (ver Figura 4), lo que contribuye, desde una perspectiva afectiva, a comprometerse más o menos en la realización de la tarea propuesta.

\section{Conclusiones}

A partir de lo expuesto arriba, nos parece claro, en primer lugar, que no podemos contentarnos con una conceptualización que sólo, o de forma predominante, se focalice en el nivel de la tarea, es decir, que la Motivación Escolar sea, fundamentalmente, una motivación por realizar las actividades propuestas por la Escuela como mediación del aprendizaje. En este sentido, asumimos como desafío el rescatar la dimensión de la actividad, es decir, una dimensión más general del fenómeno, a partir de la cual se da sentido a querer aprender, en este caso, los contenidos del currículum escolar.

\begin{tabular}{|l|c|c|}
\hline \multicolumn{3}{|c|}{ Atribuciones } \\
\hline & Interna & Externa \\
\hline Estable & Habilidad & $\begin{array}{c}\text { Dificultad de la } \\
\text { tarea }\end{array}$ \\
\hline Inestable & Esfuerzo & Suerte \\
\hline
\end{tabular}

Figura 4: Atribuciones por tipo de locus y estabilidad.

Evidentemente nos debiera interesar aquello que dispone al alumno a realizar las tareas de la clase, pero a la vez, es claro que más importante aún es no olvidar que para que un contenido pueda ser aprendido por el alumno de manera significativa y profunda, debe tener sentido aprenderla. Así, el rescatar el nivel de la actividad en la Motivación Escolar, nos resitúa en lo fundamental, a saber, la búsqueda de las claves de significación que hacen al alumno querer aprender lo que la Escuela les propone para su aprendizaje.

Creemos, finalmente, que integrar esta perspectiva y tomar en serio variables tales como la concepción de los alumnos sobre el objetivo de la Escuela, el Sentido que ellos atribuyen al Aprendizaje Escolar, la autoeficacia frente a la dinámica de aprendizaje en la Escuela y la concepción de inteligencia, puede brindar a los docentes pistas importantes para favorecer las condiciones que sus alumnos quieran aprender de manera significativa y profunda. Así, en la medida que nos preocupemos de las disposiciones relacionadas con el aprender sin dejar de lado aquellas que se vinculan con realizar las actividades de clase, estaremos en mejor pie para ayudar a nuestros alumnos a realizar más y mejores aprendizajes. 


\section{Referencias bibliográficas}

AINZENCANG, N. Los procesos de aprendizaje en contextos escolares: particularidades, problemas y desafíos. In: ELICHIRY N. (Comp.) ¿Dónde y cómo se aprende?: temas de Psicología Educacional. Buenos Aires: Eudeba, 2001, p. 11-22.

ALONSO TAPIA, J. Atribución de causalidad y motivación de logro desde una perspectiva evolutiva: evidencia empírica. Infancia y Aprendizaje, n. 26, p. 31-46, 1984.

. Análisis de las relaciones entre motivación de logro, estilos atributivos y expectativas de control a partir del estudio de la validez de constructo de los cuestionarios MAPE, EAT y ECO. Estudios de Psicología, n. 30, p. 45-69, 1987.

Motivar para el aprendizaje: teoría y estrategias. Barcelona: Edebé, 1997.

Motivación y aprendizaje en el aula: cómo enseñar a pensar. Madrid: Santillana, 2002.

ARCHAMBAULT, J.; CHOUINARD, R. Vers une gestion éducative de la classe. Montréal: Morin, 1996.

ATKINSON, J. W. Motivational determinants of risk taking behavior. Psychological Review, n. 64, p. 359-372, 1957.

AUSUBEL, D. P. Educational psychology: a cognitive view. New York: Rinehart \& Winston, 1978.

BANDURA, A. Self-efficacy: toward unifying theory of behavior change. Psychological Review, n. 84, p. 191-215, 1977.

The self-system in reciprocal determinism. American Psychologist, n. 33, p. 344-358, 1978.

Self-efficacy mechanism human agency. American Psychologist, n.37, p.122-147, 1982.

Social foundations of thought and action. New York: Prentice-Hall.1986.

Self-regulation of motivation and action through goal systems. In: HAMILTON, V; BOWER, G. H; FRIDJDA N. H. (Eds.). Cognitive perspectives on emotion and motivation. Dordrecht, Holanda: Kluver, 1988, p. 37-61. 1989.

Regulation of cognitive processes through Perceived Self-Efficacy. Developmental Psychology. v. 25, n. 5, p. 729-735,

BANDURA, A. et al. Multifaceted impact of self-efficacy beliefs on academic functioning. Child Development, v. 67, n. 3, p. 12061222, 1996.

BEAS, J. ¿Qué es un pensamiento de buena calidad? Estado de avance de la discusión. Pensamiento Educativo, n. 15, p. 13-28, 1994.

BEAS, J. et al. Enseñar a pensar para aprender mejor. Santiago: Ediciones Pontificia Universidad Católica de Chile, 2001.

Capacitar monitores para enseñar a pensar: problemas y desafíos. In: Encuentro Nacional sobre enfoques cognitivos actuales en educación. 3. Actas... Santiago: Pontificia Universidad Católica de Chile, 1996, p. 57-78.

Enseñar para la comprensión profunda: diseño y contratación de un modelo centrado en el aprendizaje y el pensamiento. Informe Final Proyecto Fondecyt n. 1950805. Santiago: Facultad de Educación, Pontificia Universidad Católica de Chile, 1997.

BRATEN, I.; STRØMS $\emptyset, H$. Epistemological beliefs and implicit theories of intelligence as predictors of achievement goals. Contemporary Educational Psychology, n. 29, p. 371-388, 2004.

BUENO, J. A. La motivación en alumnos de bajo rendimiento académico: desarrollo y programas de intervención. 1993. Tesis (Doctoral)- Universidad Complutense. Madrid, 1993.

La motivación del alumno en el aula. Madrid: ICCE, 2004.

BYRNES, J. P. Cognitive development and learning in instructional contexts. Needham Heights, Mass.: Allyn \& Bacon, 1996. 
CAIN, K.; DWECK, C. The relation between motivational patterns and achievement cognitions through the elementary school years. Merril Palmer Quaterly, v. 41, n.1, p. 25-52, 1995.

DECl, E.; RYAN, R. M. Intrinsic motivation and self-determination in human behavior. New York: Plenum, 1985. . A meta-analytic review of experiments examining the effects of extrinsic rewards on intrinsic motivation. Psychological Bulletin, v. 125, n. 6, p. 627-668, 1999.

DECI, E.; KOESTNER, R.; RYAN, R. M. Extrinsic rewards and intrinsic motivation in education: reconsidered once again. Review of Educational Research, v. 71, p. 1-27, 2001.

DECI, E. et al. Motivation and education: the self-determination perspective. Educational Psychologist, n. 26, p. 325-346, 1991.

DUPEYRAT, C.; MARINÉ, C. Implicit theories of intelligence, goal orientation, cognitive engagement, and achievement: a test of Dweck's model with returning to school adults. Contemporary Educational Psychology, n. 30, p. 43-59, 2005.

DWECK, C. Motivational processes affecting learning. American Psychologist, n. 41, p. 1040-1048, 1986.

DWECK, C.; LEGGETT, E. A social-cognitive approach to motivation and personality. Psychological Review, n. 95, p. 256-273, 1988.

ECCLES J. S. et al. Expectancies, values, and academic behaviors. In: SPENCE, J. T. (Ed.). Achievement and achievement motivation. San Francisco, CA: W. H. Freeman, 1983, p. 75-146.

ECCLES, J.; WIGFIELD, A. Motivational beliefs, values and goals. In: FISKE, S. T.; SCHACTER, D. L.; SAHN-WAXLER, C. (Eds.). Annual Review of Psychology. Palo Alto, CA: Annual Reviews, 2002, p. 109-132.

ELICHIRY, N. E. ¿¿Dónde y cómo se aprende? Buenos Aires: Eudeba, 2001.

FINDLEY, M.; COOPER, H. Locus of control and academic achievement: a literature review. Journal of Personality and Social Psychology, n. 64, p. 419-427, 1983.

FORD, M. Motivating humans: goals, emotions, and personal agency beliefs. Newbury Park, Ca: Sage, 1992.

GARCÍA, E. La naturaleza del conocimiento escolar: ¿transición de lo cotidiano a lo científico o de lo simple a lo complejo? In: RODRIGO, M. J. et al. La construcción del conocimiento escolar. Barcelona: Paidós, 1997, p. 59-79.

GLASSMAN, M. Understanding Vygotsky's motive and goal: an exploration of the work of A. N. Leontiev. Human Development, n. 39, p. 309-327, 1996.

GODEFROID, J. Psychologie science humaine. Montreal: Éditions HRW, 1991.

HANSFORD, B. C.; HATTIE, J. A. The relationship between self and achievement performance measures. Review of Educational Research, v. 52, n. 1, p. 123-142, 1982.

HEIDEGGER, M. Ser y tiempo. México: Fondo de Cultura Económica, 1971.

HUART, T. Un éclairage théorique sur la motivation scolaire: un concept éclaté en multiples facettes. Cahiers du Service de Pédagogie expérimentale, Université de Liège, n. 7-8, p. 221-240, 2001.

HUERTAS, J. A. Motivación: querer aprender. Buenos Aires: Aique, 1997.

KOZULIN, A. The concept of activity in Soviet Psychology. American Psychologist, v. 41, n. 3, p. 264-274, 1986.

LEGENDRE, R. Dictionnaire actuel de l'éducation. Guérin, Montreal, 1993.

LEONTIEV, A. Actividad, conciencia, personalidad. Buenos Aires: Edit. Ciencias del Hombre, 1978.

LOCKE, E. A.; LATHAM, G. P. A theory of goal setting and task performance. Englewood Cliffs, NJ: Prentice-Hall, 1990. 
MANASSERO, M. A.; VÁSQUEZ, A. Validación de una escala de motivación basada en la teoría atribucional de Weiner. Psicothema, v. 10, n. 2, p. 217-235, 1996.

Análisis empírico de dos escalas de motivación escolar. Revista Electrónica de Motivación y Emoción, v. 3, n. 5-6, 2000. Disponible en: <http://reme.uji.es/reme/numero5-6/indexsp.html>. Consultada el: 27 de noviembre de 2005.

MARQUIINEZ, G. Metafísica desde latinoamérica. Bogotá: Universidad Santo Tomás, 1980.

MATEOS, P. Teorías motivacionales. In: PALMERO, F. et al. (Coord.). Psicología de la motivación y la emoción. Madrid: McGraw Hill, 2002, p. 155-186.

NEUVILLE, S. La perception de la valeur des activités d'apprentissage: étude des déterminants et effets. 2004. Tesis (Doctorado)- Fac. Psychologie et des Sciences de l'Education. Université Catholique de Louvain, Louvain la Neuve. 2004. (no publicada)

NEUVILLE, S.; BOURGEOIS, É.; FRENAY, M. The subjective task value: clarification of a construct (artículo sometido). 2007.

NICHOLLS, J. Conceptions of ability and achievement motivation: a theory and its implications for education. In: PARIS, S.; OLSEN, G.; STEVENSON, H. (Eds.). Learning and motivation in the classroom. Hillsdale, NJ: Erlbaum, 1983, p. 211-237.

NUTTIN, J. Théorie de la motivation humaine: du besoin au projet d'action. Paris: Presses Universitaires de France, 1980

PARMENTIER, PH. La volonté d'apprendre. Exposición presentada al IPM. Université Catholique de Louvain. Louvain-la-Neuve, 1999. Disponible en: <www.ipm.ucl.ac.be/articlesetsupportsIPM/volonte.pdf>. Consultada en: 15/04/2006.

PERKINS, D. Smart school. New York: The Free Press; Mc Millan, 1992.

PINTOR-RAMOS, A. Realidad y sentido: desde una inspiración zubiriana. Salamanca: Publicaciones Universidad Pontificia de Salamanca, 1993.

POZO, J. I. Adquisición del conocimiento. Madrid: Morata, 2003.

RAYNAL, F. ; RIEUNIER, A. Pédagogie: dictionnaire des concepts clés. Paris: ESF, 1997.

ROCHEX, J-Y. Le sens de l'experience scolaire: entre activité et subjetivité. Paris: Presses Universitaires de France, 1998.

ROSAS, R.; BOETTO, C.; JORDÁN, V. Introducciónón a la psicología de la inteligencia. Santiago: Ediciones Universidad Católica de Chile, 1999.

RYAN, R.; DECl, E. Intrinsic and extrinsic motivations: classic definitions and new directions. Contemporary Educational Psychology, n. 25, p. 54-67, 2000

SANSONE, C.; HARACKIEWIECZ; J. Intrinsic and extrinsic motivation: the search for optimal motivation and performance. San Diego: Academic Press, 2000.

SCHIEFELE, U.; KRAPP, A.; WINTELER, A. Interest as predictor of academic achievement: a meta-analysis of research. In: RENNINGER, K. A.; HIDI, S.; KRAPP, A. (Eds.). The role of interets in learning and development. Hillsdale, NJ: Erbaum, 1992 p. $183-212$.

SCHUNK, D. Self-efficacy perspectives on achievement behaviour. Educational Psychologist, n. 19, p. 48-58, 1984.

Teorías del aprendizaje. México: Pearson, 1997.

SOMUNCUOGLU, Y.; YILDIRIM, A. Relationship between achievement goal orientations and use of learning strategies. The Journal of Educational Research, v. 92, n. 5, p. 267-277, 1999.

SUAREZ, J. M.; GONZÁLEZ, R.; VALLE, A. Multiple-goal pursuit and its relation to cognitive, self-regulatory, and motivational strategies. British Journal of Educational Psychology, n. 71, p. 561-572, 2001. 
TARDIF, J. Pour un enseignement stratégique. Montreal: Éditions Logiques, 1992.

TISHMAN, S.; JAY, E.; PERKINS, D. Teaching thinking dispositions: from transmission to enculturation. Theory into Practice, n. 32, p. $147-153,1993$.

TOBIAS, S. Interest, prior knowledge and learning. Review of Educational Research, v. 64, n. 1, p. 37-54, 1994.

TURNER, J.; MEYER, D.; SCHWEINLE, A. The importance of emotion in theories of motivation: empirical, methodological, and theoretical considerations from a goal theory perspective. International Journal of Educational Research, n. 39, p. 375-393, 2003.

UGARTETXEA, J. La metacognición, el desarrollo de la Autoeficacia y la motivación escolar. Revista de Psicodidáctica, v. 13, 2002. Disponible en: <http://148.215.4.212/rev/175/ 17501304.pdf>. Consultada en: 10 de abril de 2004.

VALENZUELA, J. Le sens de l'apprentissage scolaire: une proposition de classification des sens d'apprendre ce que l'école veut enseigner. (Mémoire de DEA) Faculté de Psychologie et des Sciences de l'Éducation. Université Catholique de Louvain, 2002.

VIAU, R. La motivation en contexte scolaire. Bruxelles: De Boeck, 1997.

WEINER, B. An atribucional theory of motivation and emotion. New York: Springer-Verlag, 1986a.

Attribution, emotion and action. In: SORRENTINO R.M.; HIGGINS, E.T. (Eds.). Handbook of motivation and cognition: foundations of social behavior NY: Guilford Press. 1986b. p. 281-312.

WENTZEL, K. R. Social and academic goals, standards for performance, and academic achievement: an integrationist perspective. Journal of Education Psychology, n. 81, p. 131-142, 1991.

WIGFIELD, A. Expectancy-value theory of achievement motivation: a developmental perspective. Educational Psychology Review, n. 6, p. 49-78, 1994.

WIGFIELD, A.; ECCLES, J. The development of achievement task values: a theoretical analysis. Developmental Review, n. 12, p. 265310, 1992.

. Expectancy-value theory of achievement motivation. Contemporary Educational Psychology, n. 25, p. 68-81, 2000.

WILLINGHAM, W.; POLACK, J.; LEWIS, C. Grades and test scores: accounting for observed differences. Journal of Educational Measurement. v. 39, p. 1-17, 2002.

ZUBIRI, X. Sobre la esencia. Madrid: Sociedad de Estudios y Publicaciones, 1962.

ZIMMERMAN, B. J. Self-Efficacy: an essential motive to learn. Contemporary Educational Psychology, n. 25, p. 82-91, 2000.

Recibido en 11.05 .06

Aprobado en 07.08.07

Jorge Valenzuela Carreño es D.E.A. en Sciences de l'Education (Université Catholique de Louvain) y Doctor en Ciencias de la Educación (Universidad Católica). 